\title{
The airway epithelium in health and disease: "calm on the surface, paddling furiously underneath"
}

\section{Rosalind L Smyth}

The airway epithelium, which is exposed to antigens and microbes throughout respiration, provides an enormous and delicate interface with the external environment. In major conducting airways the epithelium is covered by surface liquid, which includes an overlying mucous layer onto which inhaled particles are trapped. The physical defence mechanisms of the airway epithelium include the mucociliary escalator and complex structures known as tight junctions which control paracellular transport of inhaled material. In response to immunological challenge, epithelial cell surfaces produce antimicrobial chemicals (such as surfactants and defensins), cytokines (particularly type I and type III interferons) and chemokines following activation of their pattern recognition receptors, including Toll-like receptors (TLRs). This leads to recruitment of immune cells including dendritic cells, T cells and B cells.

Given the speed, complexity and specificity of these responses, it is likely that, in an environment full of antigens such as the lung, epithelial surfaces will have developed mechanisms to inhibit inflammation, as has already been demonstrated for the microbe-rich milieu of the gut. ${ }^{1}$ Although it has been recognised for some time that the airway epithelium plays an important role in generating danger signals upon immunological challenge, we need to update these concepts to recognise the importance of this interface in protecting the host from unwanted responses to innocuous environmental agents. Data are now emerging which suggest that, in health, airway epithelial cells (AECs) play a critical role in regulating local immunological homeostasis. The study by Wang et al published in this issue of Thorax provides an important contribution to this body of evidence (see page 283). ${ }^{2}$

Correspondence to: Professor R L Smyth, Division of Child Health, School of Reproductive and Developmental Medicine, University of Liverpool, Alder Hey Children's Hospital, Liverpool L12 2AP, UK; r.I.smyth@liv.ac.uk
The diverse array of receptors, including TLRs, present on AECs enables them to respond to incoming allergens, microorganisms, particulate material and initiate appropriate immunological responses. However, it is clear that TLRs cannot distinguish between pathogenic and harmless (but nonetheless immunogenic) stimuli. To protect the host from premature death due to chronic airway inflammation, other mechanisms must be invoked. These might include the ability of epithelial cells to control pattern recognition receptor sensitivity and organ-specific mechanisms to regulate immunity. ${ }^{3}$ There is some evidence for the first of these regulatory processes in the airways and the gut, where epithelial cells demonstrate ligand-dependent differences in TLR activation which correlate with differences in responses to whole organisms. ${ }^{45}$ Regulatory processes downstream of TLR activation also operate differently in different organs, suppressing inflammation in the lungs compared with organs such as the spleen where the environment is normally sterile. ${ }^{6}$

In addressing organ-specific regulation of immune responses, the study by Wang et al showed that dendritic cell-induced T cell proliferation was profoundly inhibited in a dose-dependent manner by co-culture with AECs. Experiments suggested that these effects of AECs were predominantly dependent on cell-to-cell contact, with limited effect of soluble factors, and seemed to be partially mediated by transforming growth factor $\beta$ (TGF $\beta$ ). Importantly, the experiments showed that the AECs acted directly on the T cells. Another recent study by Mayer et al which has addressed these regulatory processes showed that human bronchial epithelial cells inhibited secretion of tumour necrosis factor $\alpha(\mathrm{TNF} \alpha)$ and interleukin-12 (IL12) by monocytes, macrophages and dendritic cells, and transfer of supernatant from epithelial cell cultures caused almost as much inhibition as co-culture of cells. Mayer et al, in similar experiments to those of Wang et al, demonstrated a direct inhibitory effect of AECs on T lymphocyte proliferation, partly mediated by secretion of TGF $\beta$.

This regulation of the proinflammatory capacity of innate immune cells is much more than induction of passive "anergy". While in the experiments by Wang et al coculture inhibited the production of many $\mathrm{T}$ cell cytokines (interferon (IFN) $\gamma$, IL5, IL17, $\mathrm{TNF} \alpha$ ), it increased the production of others (granulocyte-macrophage colony stimulating factor (GM-CSF), thymic stromal lymphopoietin (TSLP)). TSLP is a novel, epithelial derived cytokine, which induces dendritic cell mediated Th2 differentiation. Mayer et al showed increased expression of arginase-1 (a marker enzyme of alternative activation by dendritic cells) and IL10 by epithelial cell-conditioned dendritic cells. Both reports also showed that the inhibitory effects of AECs were associated with an increase in the proportion of regulatory $T$ cells, including $T r 1$ and Foxp3 $^{+}$, and increased numbers of Th17 cells. Taken together, these two studies show that, in the lung-as in the gut ${ }^{1}$ organ-specific stromal cells regulate the local immune response.

With this profound downregulation of inflammatory responses by epithelial cells, how are the innate immune cells able to respond appropriately to infection? Both groups tested this, but in different ways. Mayer et al showed slightly downregulated secretion of TGF $\beta$ in epithelial cells stimulated with Pseudomonas aeruginosa. Respiratory viruses, which are major triggers of airway inflammation, primarily target AECs for infection. Wang et al showed that infection of epithelial cells with respiratory syncytial virus $24 \mathrm{~h}$ before addition of the dendritic cell/ $\mathrm{T}$ cell co-culture reduced $\mathrm{T}$ cell inhibition in proportion to the infectious dose. This effect could, in part, be mimicked by some TLR ligands (TLR3 and TLR4, but not TLR9), which suggested that, during viral infection of AECs, it is activation of these pathways that reduces their inhibition of $\mathrm{T}$ cells. Following viral infection, AECs produce type I interferons which induce a range of antiviral proteins which limit viral replication and induce AEC apoptosis. In RSV bronchiolitis there is extensive apoptosis and necrosis of AECs which slough off and contribute to small airways obstruction. Wang et al suggest that epithelial cell destruction causes not only physical problems, but further contributes to airway inflammation by releasing the 
epithelial cell layer from its immunoregulatory capacity.

What are the implications of these findings for the understanding of immune responses to respiratory viral infections in human disease? We know that, in the airways of infants with respiratory syncytial virus bronchiolitis, there is massive production of proinflammatory cytokines $^{89}$ and chemokines ${ }^{1011}$ and recruitment of inflammatory cells. ${ }^{12}$ It is difficult in clinical studies which use bronchoalveolar lavage to assess the relative contribution of AECs and recruited inflammatory cells to cytokine and chemokine production in the airways. However, indirect evidence, such as comparing the amounts of mRNA for these mediators in the cell pellet (which does not contain viable epithelial cells) with protein detected in the supernatant, suggests that even several days after the initiation of lower airway infection, AECs are actively producing inflammatory mediators. ${ }^{11}$ It is not clear whether the massive inflammation observed in the airways of infants with viral bronchiolitis represents an appropriate and necessary response to infection, or whether it signifies local immune dysregulation which enhances disease in these infants. ${ }^{13}$

Dysregulation of local immune responses following respiratory viral infection has been shown in asthma. Exacerbations of asthma are frequently initiated by common cold viruses, most commonly rhinoviruses. Infection of asthmatic AECs with rhinoviruses, compared to normal AECs, has shown that the former have limited ability to produce
IFN $\beta$ and IFN $\gamma \cdot{ }^{14}{ }^{15}$ This results in impaired apoptotic responses and increased viral replication which was reversed by exogenous IFN $\beta .{ }^{14}$ This type I interferon deficiency is highly correlated with severity of asthma exacerbations. ${ }^{15}$

The work of these two groups will lead to revisions of current concepts and provide important pointers for further research into the role of AECs in local immune regulation. These investigators have correctly redirected our attention to what happens in normal healthy epithelial cells so that we can understand better the consequences of releasing professional immune cells from inhibition by AECs. If - as the work by Mayer et al suggeststhese findings are replicated in human cells, in diseases such as asthma is there dysregulation of the homeostatic responses? Do aberrations in the delicate homeostatic control mechanisms result in over-exuberant inflammatory responses to infection which manifest as severe viral bronchiolitis or pneumonia? Is there maturation of these responses in early life when respiratory viral infections are frequent and symptomatic? We are beginning to understand that, in health, the airway epithelium is like a duck-calm on the surface but paddling furiously underneath.

Competing interests: None.

Thorax 2009;64:277-278. doi:10.1136/thx.2008.099630

\section{REFERENCES}

1. Cruickshank SM, McVay LD, Baumgart DC, et al. Colonic epithelial cell mediated suppression of CD4 T cell activation. Gut 2004:53:678-84.
2. Wang H, Su Z, Schwarze J. Healthy, but not RSVinfected, lung epithelial cells profoundly inhibit T-cell activation. Thorax 2009;64:283-90.

3. Raz E. Organ-specific regulation of innate immunity Nat Immunol 2007;8:3-4.

4. Mayer AK, Muehmer M, Mages J, et al. Differential recognition of TLR-dependent microbial ligands in human bronchial epithelial cells. J Immunol 2007:178:3134-42.

5. Melmed G, Thomas LS, Lee N, et al. Human intestinal epithelial cells are broadly unresponsive to Toll-like receptor 2-dependent bacterial ligands: implications for host-microbial interactions in the gut. $\mathrm{J}$ Immunol 2003;170:1406-15.

6. Hayashi T, Beck L, Rossetto C, et al. Inhibition of experimental asthma by indoleamine 2,3-dioxygenase. J Clin Invest 2004;114:270-9.

7. Mayer AK, Bartz H, Fey F, et al. Airway epithelial cells modify immune responses by inducing an antiinflammatory microenvironment. Eur J Immunol 2008;38:1689-99.

8. McNamara PS, Flanagan BF, Selby AM, et al. Proand anti-inflammatory responses in respiratory syncytial virus bronchiolitis. Eur Respir $J$ 2004;23:106-12.

9. McNamara PS, Flanagan BF, Baldwin LM, et al. Interleukin 9 production in the lungs of infants with severe respiratory syncytial virus bronchiolitis. Lancet 2004;363:1031-7.

10. Fletcher JN, Smyth RL, Thomas HM, et al. Respiratory syncytial virus genotypes and disease severity among children in hospital. Arch Dis Child 1997; 77:508-11.

11. McNamara PS, Flanagan BF, Hart CA, et al Production of chemokines in the lungs of infants with severe respiratory syncytial virus bronchiolitis. J Infect Dis 2005;191:1225-32.

12. McNamara PS, Ritson P, Selby A, et al Bronchoalveolar lavage cellularity in infants with severe respiratory syncytial virus bronchiolitis. Arch Dis Child 2003;88:922-6.

13. McNamara PS, Smyth RL. The pathogenesis of respiratory syncytial virus disease in childhood. $\mathrm{Br}$ Med Bull 2002;61:13-28.

14. Wark PA, Johnston SL, Bucchieri F, et al. Asthmatic bronchial epithelial cells have a deficient innate immune response to infection with rhinovirus. J Exp Med 2005;201:937-47.

15. Contoli M, Message SD, Laza-Stanca V, et al. Role of deficient type III interferon-lambda production in asthma exacerbations. Nat Med 2006:12:1023-6.

\section{Human lung mast cell heterogeneity}

\section{Peter Bradding}

Mast cells are found throughout the human body, both at mucosal surfaces and in the connective tissue of all organs. In fact, they are present in all classes of vertebrates including amphibians, reptiles, birds and mammals, and it has been estimated that the storage of histamine in vertebrate mast cells and its use as an inflammatory mediator was established in

Correspondence to: Professor P Bradding, Department of Respiratory Medicine, Glenfield Hospital, Groby Road, Leicester LE3 9QP, UK; pbradding@hotmail.com primitive reptiles (Lepidosauria) about 276 million years ago. ${ }^{1}$ This suggests that they mediate functions essential for life rather than representing a vestigial remnant of the immune system. Indeed, mast cells have been implicated in the regulation of many diverse homeostatic and pathological processes. For example, potential beneficial activities include defence against microbial infection with activation of both innate and acquired arms of the immune system, resistance to the effects of toxic venoms and roles in wound healing. ${ }^{2}$ Conversely, they are implicated in the pathophysiology of many diverse diseases such as atherosclerosis, ${ }^{4}$ allergy and asthma, ${ }^{5}$ pulmonary fibrosis ${ }^{6}$ and rheumatoid arthritis. ${ }^{7}$

Mast cells are able to achieve these diverse tissue effects through the release of numerous vasoactive and bronchospastic autacoid mediators (histamine, leukotriene C4 (LTC4), prostaglandin $\mathrm{D}_{2}$ $\left(\mathrm{PGD}_{2}\right)$, serine proteases (tryptase, chymase), cytokines and chemokines. ${ }^{2}$ The release of these mediators is not "all-ornothing", but the pattern of molecules released is often tailored to the tissue insult. For example, using gene arrays, Okumura and colleagues showed that activation of cord blood-derived mast cells with anti-IgE, lipopolysaccharide (LPS), LPS + interferon $\gamma$ (IFN $\gamma$ ) or IFN $\gamma$ alone induced both core responses but also 